\title{
Assessing dose contribution to pelvic lymph nodes in intracavitary brachytherapy for cervical cancer
}

\author{
Gail Wan Ying Chua, BM BCh, Yong Wee Foo, MSc, CMD, Guan Heng Tay, BSc, David Boon Harn Tan, MBBS, FRCR \\ Division of Radiation Oncology, National Cancer Centre Singapore
}

\begin{abstract}
Purpose: In radical radiotherapy for cervical cancer, high-dose-rate (HDR) brachytherapy is commonly used after external beam radiation therapy (EBRT) to deliver a cumulative $\mathrm{EQD}_{2}$ of 80 to 90 Gy to the primary tumor. However, there is less certainty regarding brachytherapy dose contribution to the pelvic lymph nodes. This poses a challenge as to how high a preceding EBRT dose should be prescribed to gross nodal disease, in order to achieve a cumulative tumoricidal effect. Hence, this study aims to quantify brachytherapy dose contribution to individual pelvic nodal groups, using computed tomography (CT) planning with the Manchester system.

Material and methods: This is a single institution retrospective dosimetric study. CT planning datasets from 40 patients who received EBRT followed by intracavitary HDR brachytherapy (5 or 6 Gy fractions) were retrieved. The external iliac (EI), internal iliac (II), and obturator (OB) lymph node groups were contoured on each CT dataset. Applying the initial brachytherapy plan, mean doses to each nodal group were calculated for every patient, and averaged across the respective ( 5 or 6 Gy) study populations.

Results: With a brachytherapy dose of $5 \mathrm{~Gy}$ to Manchester point A, the mean absolute doses received by the EI, II, and $\mathrm{OB}$ groups were $0.79,1.12$, and $1.34 \mathrm{~Gy}$ respectively, corresponding to $\mathrm{EQD}_{2 \mathrm{~s}}(\alpha / \beta=10)$ of $0.71,1.04$, and $1.27 \mathrm{~Gy}$ respectively. With a brachytherapy dose of $6 \mathrm{~Gy}$, the mean absolute doses received by the EI, II, and OB groups were $1.16,1.56$, and 1.80 Gy respectively, corresponding to $\mathrm{EQD}_{2 \mathrm{~s}}$ of $1.08,1.49$, and $1.77 \mathrm{~Gy}$, respectively.

Conclusions: Our study demonstrates that pelvic lymph nodes receive substantial dose contributions from HDR brachytherapy in cervical cancer. This should be taken into account by the radiation oncologist during EBRT planning, and adequate external beam boost doses calculated to achieve cumulative tumoricidal doses to pelvic nodal disease.

Key words: brachytherapy, cervical cancer, dosimetry, pelvis.

\section{Purpose}

In definitive radiotherapy for cervical cancer, a highdose-rate (HDR) brachytherapy boost is most commonly used after external beam radiation (EBRT). While brachytherapy doses are chosen such that a cumulative $\mathrm{EQD}_{2}$ (equivalent dose at $2 \mathrm{~Gy}$ ) of 80 to $90 \mathrm{~Gy}$ is delivered to the primary tumor after a 45 to 50.4 Gy EBRT dose, there is less certainty regarding brachytherapy dose contribution to pelvic lymph nodes (PLNs). This poses a challenge as to how high a preceding EBRT dose should be prescribed to gross nodal disease, in order to achieve a cumulative tumoricidal effect.

While the use of magnetic resonance imaging (MRI)guided 3-dimensional brachytherapy is increasing, and indeed offers several advantages over point-based treatment planning [1], many centers are unable to offer this due to logistical or financial considerations. Therefore, point-based 2-dimensional brachytherapy planning, in particular using the Manchester system, continues to be most widely employed across the world.

With the Manchester system, point A is defined anatomically as $2 \mathrm{~cm}$ lateral to the central uterine canal and $2 \mathrm{~cm}$ superior to the lateral fornix in the plane of the uterus. It is taken to be representative of the average dose to the tumor, with brachytherapy prescription consequently being specified to that point. Point $B$ is defined as being $3 \mathrm{~cm}$ lateral to point $A$, i.e. $5 \mathrm{~cm}$ from the midline on the lateral axis. It is also recorded during the treatment and has traditionally been thought to represent the pelvic sidewall and thus PLN dose. However, a study conducted by Lee et al. in 2009 suggested that this correlation was poor [2]. In this study, the authors investigated the correlation between point $B$ dose and the dose to the various pelvic lymph node groups. Patients with FIGO (International Federation of Gynecology and Obstetrics) stage IB-IIIB cervical cancer received 70 tandem HDR applications using computed tomography (CT)-based treatment
Address for correspondence: David Boon Harn Tan, MBBS, FRCR, Division of Radiation Oncology, National Cancer Centre, Singapore, 11 Hospital Drive, Singapore 169610, phone: +65 63214204, fax: +65 62228675, $\bowtie$ e-mail: david.tan.b.h@nccs.com.sg
Received: 27.02 .2017

Accepted: 29.05 .2017

Published: 30.08 .2017 
planning. The obturator (OB), external iliac (EI), and internal iliac (II) PLNs were contoured, and doses per fraction and combined fraction right, left, and bilateral nodal doses were analyzed. Point B dose was then compared with PLN dose-volume histogram (DVH) parameters by a paired $t$-test and Pearson correlation coefficients. Results showed that the correlation coefficients between point $\mathrm{B}$ and the $\mathrm{D}_{100}, \mathrm{D}_{90}, \mathrm{D}_{50}, \mathrm{D}_{2 \mathrm{cc}}, \mathrm{D}_{1 \mathrm{cc}}$ and $\mathrm{D}_{0.1 \mathrm{cc}}$ (percent of the prescription dose covering $100 \%, 90 \%, 50 \%$ of the clinical target volume [CTV], minimum dose to the most exposed $2 \mathrm{~cm}^{3}, 1 \mathrm{~cm}^{3}, 0.1 \mathrm{~cm}^{3}$ ) PLNs were all less than 0.7 , demonstrating a poor overall correlation [2].

Two other studies have attempted to quantify the doses to the PLNs from brachytherapy. Lee et al. aimed to investigate the variation in HDR brachytherapy dose to pathologically involved common iliac, external and internal iliac, and obturator lymph nodes [3]. Twenty-one patients with locally advanced cervical cancer were chosen, all of whom received radical external beam radiotherapy and HDR brachytherapy. These patients had a total of 45 positive PLNs. These nodes were contoured on both the external beam and brachytherapy planning images. The average total dose delivered to the positive nodes in each lymph node group was found and the corresponding $\mathrm{EQD}_{2}$ calculated. The study found a $4.1-9.5 \%$ variation in brachytherapy dose received among the different PLN groups, a statistically significant difference, with the obturator nodes receiving the highest doses [3].

Another study by Mohamed et al. evaluated the dose delivered to PLNs by MRI pulsed-dose-rate (PDR) brachytherapy [4]. This study analyzed 25 patients with locally advanced cervical cancer who received intensity-modulated radiotherapy (IMRT) (45-50 Gy in 25-30 fractions) to the whole pelvis and 2 fractions of MRI PDR brachytherapy. The delineated PLN groups were para-aortic, inguinal, common iliac, EI, II, OB, and presacral. For each PLN group, $\mathrm{D}_{98 \%}, \mathrm{D}_{50 \%}$, and $\mathrm{D}_{2 \%}$ were evaluated, and the correlation between total reference air kerma and $D_{50 \%}$ of the PLN groups was calculated. The study found that brachytherapy contributed considerable dose, the mean $\mathrm{D}_{50 \%}$ being 3.8-6.2 Gy equivalent total dose in 2 Gy fractions to the PLNs in optimized plans [4].

Meanwhile, Kamer et al. considered 5 patients with advanced cervical cancer who underwent lymph node irradiation, assessing EBRT nodal boost dose contribution to the high-risk CTV, as well as PDR brachytherapy doses to involved iliac nodes. High-risk CTV, organs at risk (OARs), and involved nodes were contoured on T2 weighted MRI, and biologically weighted $3 \mathrm{DEQD}_{2}$ dose distributions were calculated for the complete treatment. This group found that brachytherapy dose to $D_{90}$ of the PLNs was between 3-12 Gy, highly dependent on the location of the nodes [5].

Apart from the studies above, few others have attempted to determine the actual dose contribution of intracavitary brachytherapy to pelvic lymph nodes in the treatment of cervical cancer. Moreover, of the studies discussed, two investigated mean doses to involved PLNs only, rather than the entire nodal group, while two used pulsed-dose-rate brachytherapy, which may be difficult to extrapolate to more commonly practiced continuous HDR brachytherapy treatment. Nonetheless, the data available has informed us of several important issues about doses received by PLNs during brachytherapy: 1) they correlate poorly with traditionally reported pointbased dose parameters; 2 ) they can be considerable (as high as 12 Gy $\mathrm{EQD}_{2}$ in one study); 3) they can be highly variable depending on the location of the PLN. It was with the above points in mind that our study has been designed. It aims to evaluate brachytherapy dose contribution to individual PLN regions (namely EI, II, and OB) in order to provide a set of dosimetric information that reflects the location of the pelvic lymph node (to the level of the individual PLN groups). This will provide a conservative reference dose that is broadly applicable for the use by the radiation oncologist to guide the radiotherapy planning process. The results of our study will also add to, and validate the results of the few previously published studies $[2,3,4,5]$ on this subject. Our study focused on patients treated with the Manchester system, as this is the most commonly utilized point-based prescription technique.

\section{Material and methods}

\section{Patient characteristics}

A retrospective analysis was performed using CT planning datasets from 40 patients with stage 1B1-4B cervical cancer, histologically confirmed by biopsy, and staged using MRI. Both adenocarcinoma and squamous cell carcinoma histology were included in the study.

Details of patients characteristics are presented in Table 1 . The mean age of study group was 54.4 . The majority were Chinese $(26 / 40)$, the others being Malay, Indian, or others (e.g. Bruneian). The most common stage of cancer was FIGO 2B (22/40), and eighteen patients were equally distributed across other stages. One patient was staged $4 \mathrm{~B}$ due to bone metastases, but was included in the study as the brachytherapy dose and technique were in accordance with our study design. 16 external iliac nodes, 4 internal iliac nodes, and 5 obturator nodes were pathologically involved, defined as being greater than $1.0 \mathrm{~cm}$ in short axis in diameter on initial staging CT and/or MRI. Of the 40 patients, 13 patients had at least 1 pathologically involved lymph node, while 6 patients had more than 1 involved node. Of these 6 patients, 2 were in the 5 Gy per fraction group, while 4 were in the 6 Gy per fraction group.

Between May 2014 and June 2015, all patients underwent radical EBRT and intracavitary HDR brachytherapy for cervical cancer. All patients were treated at the National Cancer Centre Singapore. Institutional review board approval was obtained for the retrospective analysis.

The treatment regimen was as follows: patients received 3D conformal EBRT to a dose of $50.4 \mathrm{~Gy}$ in 28 fractions, followed by 3 or 4 HDR brachytherapy fractions with CT-planning, prescribed to Manchester point A. Half of the patients $(n=20)$ received 5 Gy brachytherapy dose per fraction, while the other half received 6 Gy per fraction. Decision on brachytherapy dose was dependent on the ability to meet $\mathrm{D}_{2 \mathrm{cc}}$ constraints for the adjacent OARs, namely rectum, sig- 
Table 1. Summary of patients characteristics

\begin{tabular}{|c|c|c|c|}
\hline Factors & 5 Gy $(n=20)$ & 6 Gy $(n=20)$ & Overall $(n=40)$ \\
\hline Mean age (years) & 56.4 (range, 34-88) & 52.3 (range, 40-80) & 54.4 \\
\hline \multicolumn{4}{|l|}{ Race* $^{*}$} \\
\hline Chinese & 12 & 14 & 26 \\
\hline Malay & 6 & 3 & 9 \\
\hline Indian & 2 & 1 & 3 \\
\hline Others & 0 & 2 & 2 \\
\hline \multicolumn{4}{|c|}{ Tumour stage (FIGO) } \\
\hline 1B1 & 0 & 2 & 2 \\
\hline 1B2 & 2 & 2 & 4 \\
\hline $2 \mathrm{~A}$ & 2 & 1 & 3 \\
\hline $2 B$ & 13 & 9 & 22 \\
\hline $3 \mathrm{~A}$ & 1 & 1 & 2 \\
\hline $3 B$ & 1 & 3 & 4 \\
\hline $4 \mathrm{~A}$ & 1 & 1 & 2 \\
\hline $4 \mathrm{~B}$ & 0 & 1 & 1 \\
\hline \multicolumn{4}{|c|}{ Pathologically involved nodes } \\
\hline External iliac & 7 & 9 & 16 \\
\hline Internal iliac & 3 & 1 & 4 \\
\hline Obturator & 1 & 4 & 5 \\
\hline
\end{tabular}

moid, small bowel, and bladder. The majority of patients received concurrent chemo-radiotherapy; however, some received radical radiotherapy alone, due to factors such as advanced age or an earlier stage of disease.

\section{External beam radiation therapy}

All patients underwent $\mathrm{CT}$ simulation with localization markers. Each patient was positioned supine on a couch, with the head on a low headrest, arms at the sides, and with wedge support for the knees. The bladder was comfortably full ( 3 cups of water $30 \mathrm{~min}$ before simulation). A tampon was used to outline the vagina and location of the cervix. Contrast enema was used to highlight the rectum.

A CT scan was performed with 2 or $2.5 \mathrm{~mm}$ slices from L2/L3 superiorly to the perineum inferiorly. Target volumes were contoured using the Eclipse planning system. The clinical target volume included the cervical tumor, uterus, regional lymph nodes, upper vagina and parametria. A four field plan was used with anterior, posterior, and two lateral fields with equal weighting from each field. Average field sizes were $12-15 \mathrm{~cm}(\mathrm{~S}-\mathrm{I}) \times 14-17 \mathrm{~cm}$ for the A/P fields, and $12-15 \mathrm{~cm}(\mathrm{~S}-\mathrm{I}) \times 10-12 \mathrm{~cm}$ for the lateral fields. A dose of $50.4 \mathrm{~Gy}$ was prescribed to the ICRU reference point at the intersection of the beams. Treatment was given for 5 days a week, to a total of 28 fractions over 5.5 weeks. No central shielding was used.

\section{High-dose-rate brachytherapy}

In total, all patients underwent 3 to 4 intracavitary brachytherapy insertions with ${ }^{192} \mathrm{Ir}$. Conscious sedation with intravenous midazolam was administered, and pain control achieved with IV fentanyl. Each patient was positioned in the lithotomy position and catheterized prior to the procedure.

Examination of the tumor under sedation was carried out, and an uterine sound was used to measure the length of the uterine cavity. Fletcher-Williamson or Rotterdam tandem and ovoid applicators were used for insertions, and the applicators were then secured in place with vaginal packing. Following the insertion, a CT simulation was then performed on alternate fractions, and the rectum, bladder, sigmoid colon, and small bowel were contoured to calculate dose constraints.

Brachytherapy doses ranged from 18 Gy to 24 Gy in 3 to 4 fractions (i.e. 5-6 Gy per fraction). All doses were prescribed to point A using the Manchester system.

\section{Dosimetric analysis}

Following international consensus guidelines [6,7,8,9], the right and left EI, II, and OB groups of PLNs were separately contoured on the CT dataset for each patient in the study. The borders of our nodal contours are stated in Table 2, and a sample diagram is shown in Figure 1. 
In order to ensure consistency, the contours were independently reviewed by 2 radiation oncologists. Only the $\mathrm{CT}$ of the first fraction was used for contouring of nodal regions and dosimetric analysis, in order to account for differences in anatomy between patients.
Applying the initial brachytherapy plan on the Oncentra treatment planning system (Figures 2 and 3), the mean doses to each nodal group according to laterality - left and right - were calculated for each patient. The individual patient results were then averaged across all

Table 2. Lymph node boundaries used in our study (adapted from international consensus guidelines) $[6,7,8,9]$

\begin{tabular}{|c|c|c|c|c|c|c|}
\hline & Cranial margin & Caudal margin & Anterior margin & Posterior margin & Lateral margin & Medial margin \\
\hline External iliac & $\begin{array}{l}\text { L5/S1 - common } \\
\text { iliac (CI) bifurca- } \\
\text { tion or } 2 \mathrm{~cm} \text { above } \\
\text { top of applicator* } \\
\text { whichever is } \\
\text { inferior }\end{array}$ & $\begin{array}{c}\text { Superior femoral } \\
\text { head }\end{array}$ & $\begin{array}{l}10 \mathrm{~mm} \text { anterior } \\
\text { to vessels }\end{array}$ & $\begin{array}{l}7 \mathrm{~mm} \text { posterior } \\
\text { to vessels }\end{array}$ & $\begin{array}{c}7 \mathrm{~mm} \text { lateral } \\
\text { to vessels }\end{array}$ & $\begin{array}{l}7 \mathrm{~mm} \text { medial } \\
\text { to vessels }\end{array}$ \\
\hline Internal iliac & $\begin{array}{l}\mathrm{L} 5 / \mathrm{S} 1-\mathrm{Cl} \text { bifurca- } \\
\text { tion or } 2 \mathrm{~cm} \text { above } \\
\text { top of applicator } \\
\text { whichever is } \\
\text { inferior }\end{array}$ & $\begin{array}{l}\text { Termination } \\
\text { of internal iliac } \\
\text { vessels }\end{array}$ & $\begin{array}{l}7 \mathrm{~mm} \text { anterior } \\
\text { to vessels }\end{array}$ & $\begin{array}{c}7 \mathrm{~mm} \text { posterior } \\
\text { to vessels }\end{array}$ & $\begin{array}{l}7 \mathrm{~mm} \text { lateral } \\
\text { to vessels }\end{array}$ & $\begin{array}{l}7 \mathrm{~mm} \text { medial } \\
\text { to vessels }\end{array}$ \\
\hline Obturator & $\begin{array}{l}\text { Commencement } \\
\text { of gap between } \\
\text { external and inter- } \\
\text { nal iliac regions }\end{array}$ & $\begin{array}{l}\text { Obturator } \\
\text { foramen }\end{array}$ & $\begin{array}{l}\text { Connecting } \\
\text { to external iliac } \\
\text { region }\end{array}$ & $\begin{array}{l}\text { Connecting } \\
\text { to internal iliac } \\
\text { region }\end{array}$ & $\begin{array}{l}7 \mathrm{~mm} \text { lateral } \\
\text { to vessels }\end{array}$ & $\begin{array}{l}7 \mathrm{~mm} \text { medial } \\
\text { to vessels }\end{array}$ \\
\hline
\end{tabular}

All CTV's were shaved off bone, muscle, and pelvic organs - bladder, rectum, uterus. Any visible nodes were also included

*We standardised the superior cut-off at $2 \mathrm{~cm}$ above the applicator due to (1) limitations of the brachytherapy CT scan, and the fact that (2) dose to the lymph node regions beyond this point is likely to be negligible.

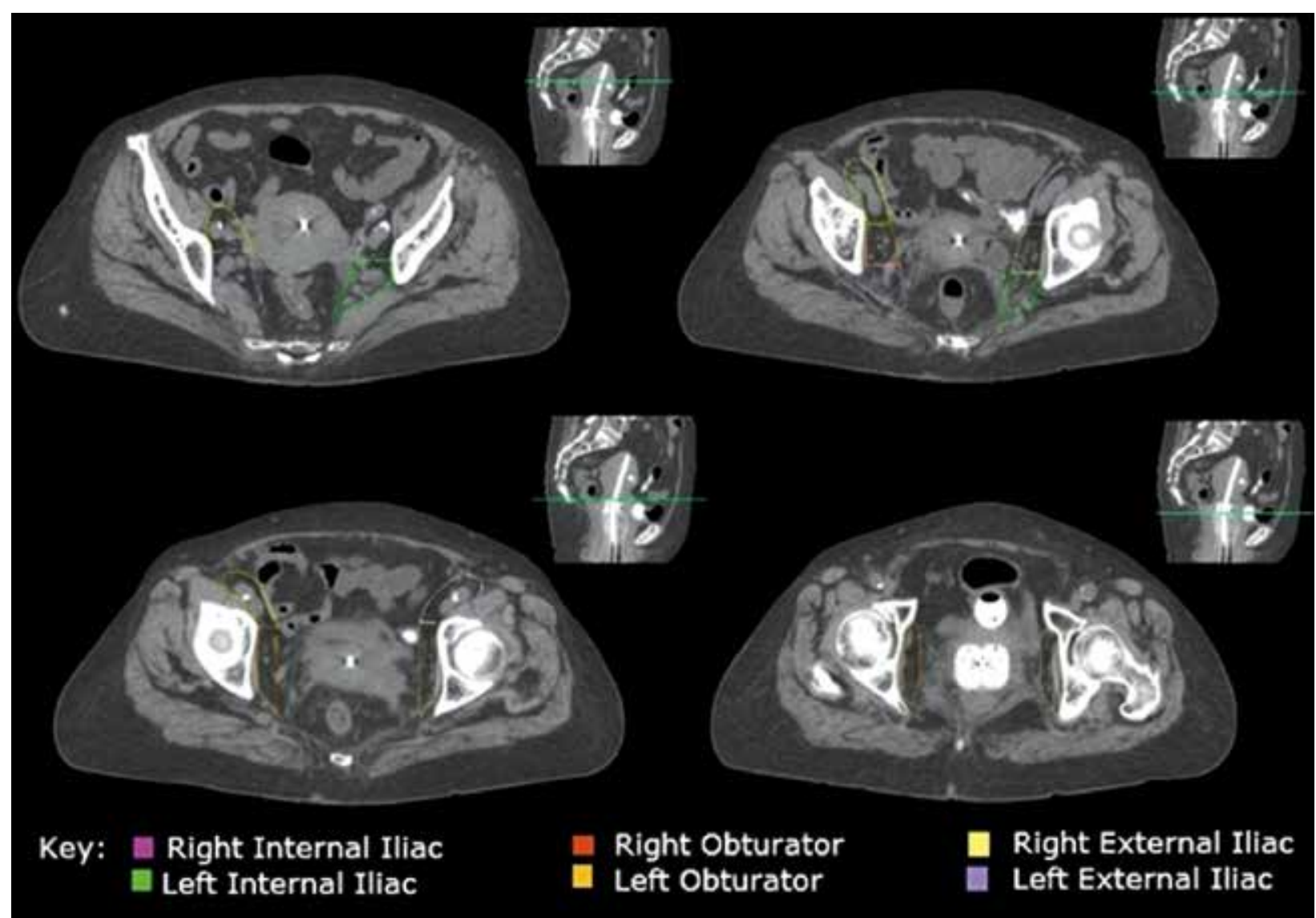

Fig. 1. Contours of the lymph node groups at 4 different levels in a single patient 

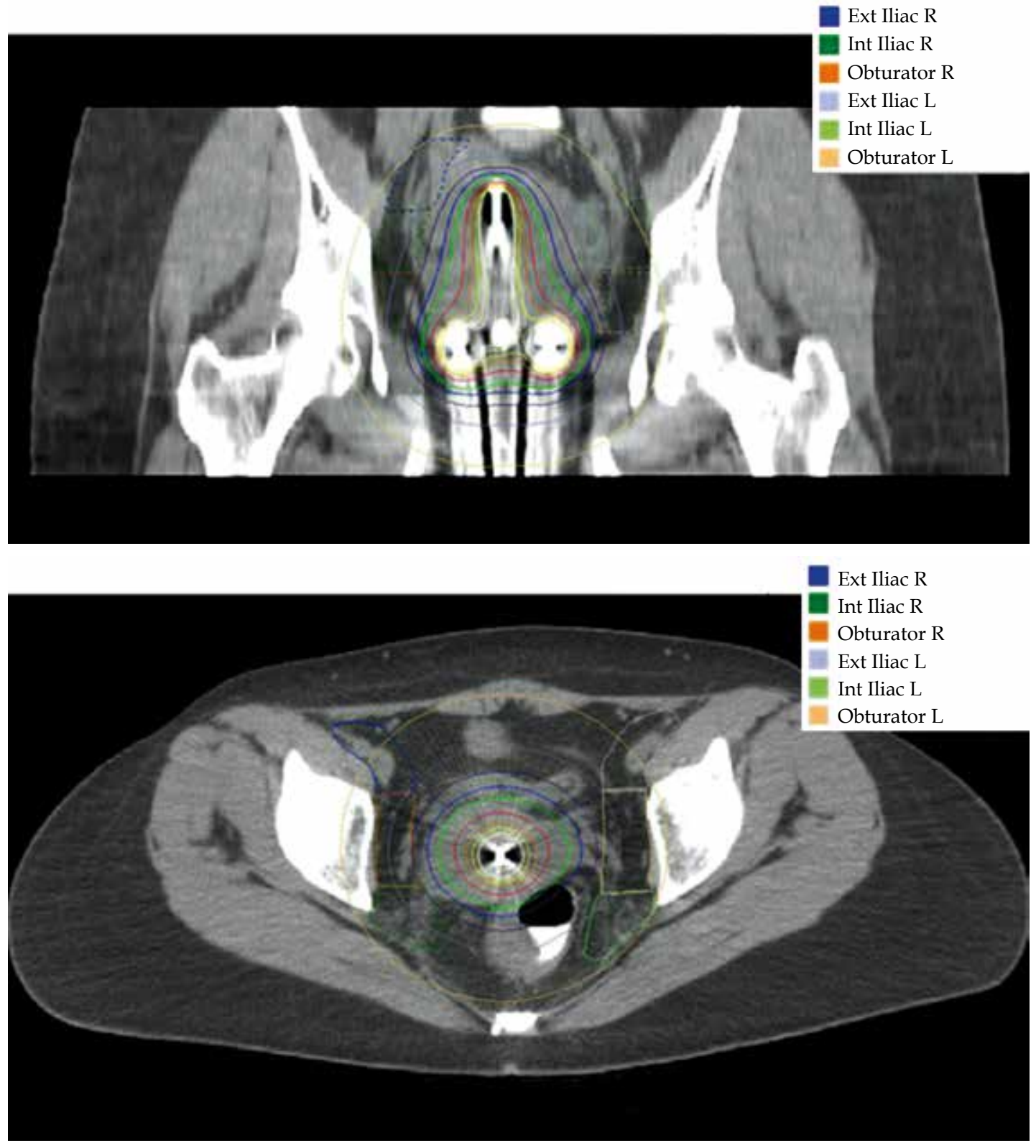

Fig. 2. Coronal and axial sections from a single patient (isodose lines are demarcated around the applicator)

patients within the respective 5 Gy or 6 Gy dose groups, and the population mean and median absolute doses to each PLN group were obtained. The corresponding EQD $2 \mathrm{~s}$ $(\alpha / \beta=10)$ for the mean doses were then calculated.

\section{Results}

\section{Brachytherapy dose to pelvic lymph nodes}

Among the 20 patients who received 5 Gy per fraction prescribed to point $\mathrm{A}$, the mean doses to the EI, II, and OB nodal groups were $0.79 \mathrm{~Gy}, 1.12 \mathrm{~Gy}$, and $1.34 \mathrm{~Gy}$ respectively, representing $15.9 \%, 22.5 \%$, and $26.9 \%$ of the prescribed dose. The corresponding $\operatorname{EQD}_{2 \mathrm{~s}}(\alpha / \beta=10)$ were $0.71 \mathrm{~Gy}, 1.04 \mathrm{~Gy}$, and $1.27 \mathrm{~Gy}$ respectively.

Among the 20 patients who received $6 \mathrm{~Gy}$ per fraction prescribed to point $\mathrm{A}$, the mean doses to the EI, II, and OB nodal groups were $1.16 \mathrm{~Gy}, 1.56 \mathrm{~Gy}$, and $1.80 \mathrm{~Gy}$ respectively, representing $19.3 \%, 25.8 \%$, and $30.1 \%$ of the prescribed dose. The corresponding $\operatorname{EQD}_{2 \mathrm{~s}}(\alpha / \beta=10)$ were $1.08 \mathrm{~Gy}, 1.49 \mathrm{~Gy}$, and 1.77 Gy respectively.

Details of our results are summarized in Table 3, and further presented in box plots in Figure 3. As seen from the box plots, the presence of outliers did not significantly influence the mean values.

\section{Discussion}

Our results demonstrate that the PLNs receive a significant dose contribution from intracavitary HDR brachytherapy in cervical cancer. Expressing the per fraction mean dose to each PLN region as a percentage of the prescribed dose (i.e. 5 or 6 Gy), we found that the 
A

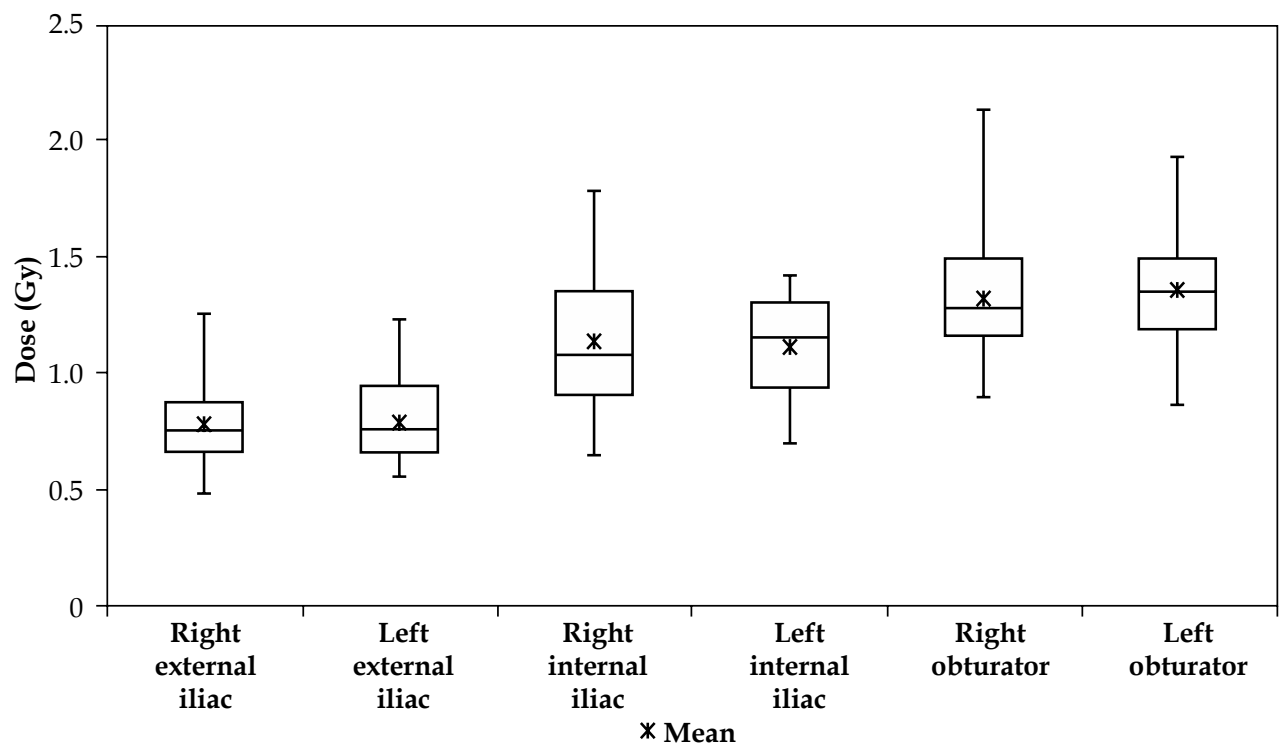

B

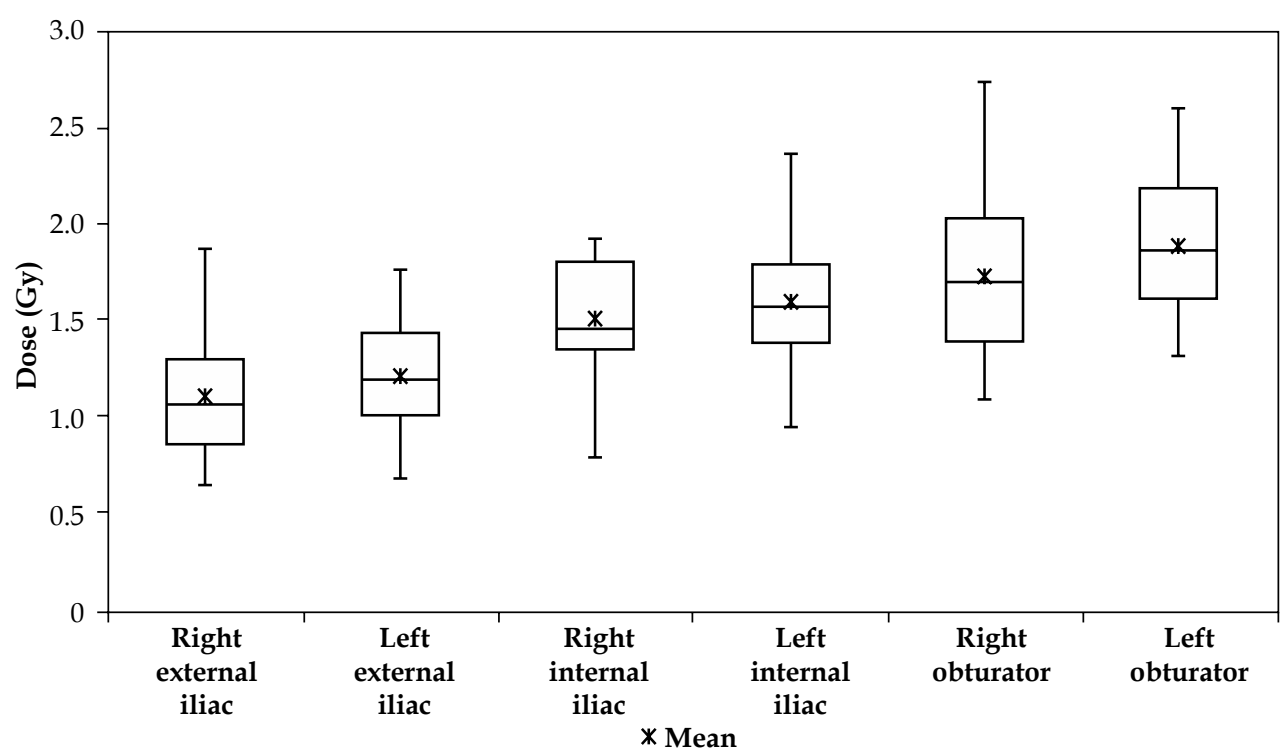

Fig. 3. Summary of doses to lymph node regions for patients: A) 5 Gy patients, B) 6 Gy patients

pelvic nodal groups, when averaged across the respective study populations, received a minimum of $15.9 \%$ and a maximum of $30.1 \%$ of the prescribed dose depending on the PLN group considered. When summated across 4 brachytherapy fractions, which is the standard in our centre, the cumulative $\mathrm{EQD}_{2}$ doses to the EI, II, and OB nodes were $2.85 \mathrm{~Gy}, 4.17 \mathrm{~Gy}$, and 5.08 Gy respectively in patients who received $5 \mathrm{~Gy} \times 4$ fractions to point $\mathrm{A}$, and $4.30 \mathrm{~Gy}, 5.95 \mathrm{~Gy}$, and 7.09 Gy respectively in patients who received $6 \mathrm{~Gy} \times 4$ fractions.

Considering that brachytherapy dose contributions to PLNs are usually thought to be negligible and not taken into account during radiotherapy planning, our results show on the contrary, these doses are substantial and should not be ignored. Clinicians may take this data as a reference, for example in planning boost doses to grossly involved pelvic lymph nodes in the nodal regions we have studied. However, there is an important caveat to the application of our results: nodal regions are anatomically complex and can include nodes that are at substantially different distances from brachytherapy sources. Due to the rapid fall-off of radiation dose with distance from the source, nodes that are closer may receive substantially higher doses than nodes that are further away, even though they are located within the same nodal region. As the mean doses we have calculated are single average values across each nodal region, the radiation oncologist must exercise clinical judgment in applying our results, always taking into account the location of the involved node in relation to the brachytherapy source. We have also presented our dosimetric results in a box plot (see Figure 3 ) to show the possible range of doses within each nodal region. We believe that this will further help the radiation oncologist to estimate a higher or lower dose to spatially different lymph nodes in the same group. 
Table 3. Per fraction brachytherapy dose contribution to different pelvic lymph node groups, expressed as a percentage of total dose, and corresponding $\mathrm{EQD}_{2}(\alpha / \beta=10)$

\begin{tabular}{|c|c|c|}
\hline Per fraction brachytherapy dose contribution & 5 Gy group $(n=20)$ & 6 Gy group $(n=20)$ \\
\hline \multicolumn{3}{|l|}{ External iliac } \\
\hline Mean absolute dose, right (median; IQR) (Gy) & $0.785(0.755 ; 0.658-0.878)$ & $1.104(1.055 ; 0.855-1.293)$ \\
\hline Mean absolute dose, left (median; IQR) (Gy) & $0.801(0.760 ; 0.660-0.940)$ & $1.208(1.190 ; 1.005-1.423)$ \\
\hline Mean absolute dose, average of right and left (Gy) & 0.793 & 1.156 \\
\hline Corresponding $\mathrm{EQD}_{2}(\mathrm{~Gy})$ & 0.713 & 1.075 \\
\hline Mean dose expressed as a percentage of prescribed dose (\%) & 15.86 & 19.27 \\
\hline \multicolumn{3}{|l|}{ Internal iliac } \\
\hline Mean absolute dose, right (median; IQR) (Gy) & $1.135(1.080 ; 0.908-1.353)$ & $1.500(1.450 ; 1.345-1.800)$ \\
\hline Mean absolute dose, left (median; IQR) (Gy) & $1.113(1.155 ; 0.938-1.308)$ & $1.593(1.560 ; 1.388-1.783)$ \\
\hline Mean absolute dose, average of right and left (Gy) & 1.124 & 1.546 \\
\hline Corresponding $\mathrm{EQD}_{2}(\mathrm{~Gy})$ & 1.042 & 1.488 \\
\hline Mean dose expressed as a percentage of prescribed dose (\%) & 22.48 & 25.77 \\
\hline \multicolumn{3}{|l|}{ Obturator } \\
\hline Mean absolute dose, right (median; IQR) (Gy) & $1.328(1.285 ; 1.163-1.493)$ & $1.722(1.690 ; 1.390-2.023)$ \\
\hline Mean absolute dose, left (median; IQR) (Gy) & 1.359 (1.350; 1.193-1.495) & $1.884(1.855 ; 1.600-2.178)$ \\
\hline Mean absolute dose, average of right and left (Gy) & 1.343 & 1.803 \\
\hline Corresponding $\mathrm{EQD}_{2}(\mathrm{~Gy})$ & 1.270 & 1.773 \\
\hline Mean dose expressed as a percentage of prescribed dose (\%) & 26.86 & 30.05 \\
\hline
\end{tabular}

$E Q D_{2}$ - equivalent dose at $2 \mathrm{~Gy}$

Several observations have also been made on further analysis of our results. First, we noted that the EI nodal group received the lowest doses, followed by the II group, and the OB group. Relatively high OB nodal doses have also been observed in a previous published study [3]. This is likely due to the fact that the OB lymph node region is situated most inferiorly and medially, and thus closest to the applicators.

Next, we observed that there were some distal outliers in our study population (see Figure 3). We identified those patients and examined their CT images and brachytherapy plans to identify possible causes for the dose variations. Specifically, we looked at the contoured PLN regions for discrepancies of contouring, spatial arrangements of adjacent organs, and pelvic girth. In particular, the interspinous and intertuberous distances, which have been used as indicators of pelvic width in prior studies [10], were measured to determine if there was any relationship between pelvic girth and brachytherapy dose to the pelvic side wall. We found that when comparing patients at both ends of the spectrum, the interspinous and intertuberous distances were significantly different. For example, among the patients prescribed 6 Gy per fraction, those receiving a right EI dose that was above the $75^{\text {th }}$ percentile had a median intertuberous and interspinous distance of $102.1 \mathrm{~mm}$ and $115.4 \mathrm{~mm}$ respectively. In contrast, those receiving a right EI dose below the $25^{\text {th }}$ percentile had a wider median intertuberous and interspinous distance of $114.3 \mathrm{~mm}$ and $126.5 \mathrm{~mm}$ respectively. This, together with the other factors that may influence pelvic sidewall dose from brachytherapy, is a subject of further exploration by our group.

In order to set our results in context, we performed a brief review of the literature concerning doses to involved PLNs in cervical cancer. In cases with positive PLNs, an external beam nodal boost is generally recommended to prevent recurrence. For example, in a study by Wakatuski et al., researchers found that for pelvic nodes that were still enlarged by size criteria after receiving a dose of $50 \mathrm{~Gy}$, radiation dose received was a good predictor of recurrence. Specifically, 9/16 nodes receiving $\leq 58$ Gy had recurrence, but $0 / 21$ nodes receiving $>58$ Gy had recurrence $(p=0.0003)$ [11]. Similarly, Grisby et al. reported clinical outcomes of 132 patients treated with EBRT boost to pelvic nodes [12]. With a mean PLN boost dose of 9.9 Gy and a total PLN dose of 67.2 Gy, only 5 patients $(4 \%)$ in the entire study group developed PLN recurrence. Other published studies have delivered an initial EBRT dose of $50 \mathrm{~Gy}$, followed by a 6-10 Gy boost administered in 3-5 fractions [13]. In these cases, treatment planning CTs were performed after the initial 50 Gy to define residual nodal disease and to plan the external beam boost. Alternatively, IMRT may be used to deliver the doses using a simultaneous integrated boost (SIB) approach, delivering doses such 
as 55-60 Gy in 2.12-2.4 Gy fractions [14]. The 2015 National Comprehensive Cancer Network (NCCN) guidelines suggest that grossly involved unresected nodes may be evaluated for a boost with an additional 10-15 Gy of highly conformal EBRT, following a definitive dose of 40-50 Gy [15].

These data certainly further illustrate the importance of our study in providing an estimate of the brachytherapy dose contribution to each PLN region, even before brachytherapy is performed. It would allow the radiation oncologist to plan an appropriate external beam boost to involved PLNs, in order to achieve a cumulative tumoricidal effect.

When calculating accumulation of doses to PLNs, we followed standard GEC-ESTRO practice, namely a straightforward addition of the DVH parameters for EBRT and brachytherapy insertions. However, it should be noted that simple addition of DVH parameters assumes that the location of the region of interest is identical in EBRT treatment, as well as brachytherapy insertions. Shouldthe high-dose area undergo a significant shift, straightforward addition of DVH parameters would no longer hold and it would be necessary to illustrate cumulative dose distributions of IMRT and brachytherapy plans, in order to calculate DVH $[16,17]$.

In prostate cancer treatment research, Hinton et al. used iliac vessels as a surrogate for PLN movement, and found that mean daily lateral and AP shifts of iliac vessels were $2.1 \mathrm{~mm}( \pm 2.2)$ and $3.5 \mathrm{~mm}( \pm 2.7)$, respectively. Hence, the authors recommended an additional PLNCTV to PLN-PTV conversion margin of $9 \mathrm{~mm}(\mathrm{AP})$ and $7 \mathrm{~mm}$ (lateral) to account for this [18]. Accordingly, we recognize that there is potential for PLN movement, both during EBRT and brachytherapy, which is not specifically quantified in the contouring guidelines we consulted $[6,7,8,9]$. However, as we are considering the nodal regions as a group, the impact on dosimetry is likely to be relatively smaller than if we were considering the pathologically involved nodes themselves.

Finally, it should be noted that though radiotherapy dose to PLN is certainly important, other factors may have an impact on tumor control as well, such as initial nodal PET-avidity and number of chemotherapy cycles received. Ramlov et al. considered 139 patients from the EMBRACE study, and determined individual nodal dose by dose maps from EBRT and IGABT. PET-CT scans were re-evaluated and the relationship between $S U V_{\max }$ and nodal control was analyzed. In general, 6 patients had recurrences in a boosted node, and $\mathrm{SUV}_{\max }$ was significantly higher in nodes that failed, showing that high nodal $\mathrm{SUV}_{\max }$ has negative prognostic predictive value for nodal control. Furthermore, the group found that patients who received 4 or fewer cycles of cisplatin had a greater incidence of nodal failure [19]. Hence, our dosimetric results should be placed in context - and while our data could help radiation oncologists in their treatment planning, attention should also be paid to giving: 1 ) an adequate dose of chemotherapy; and 2) close follow-up of patients with highly PET-avid nodes (if initial PET scans are available) for monitoring of potential nodal recurrences.
While our study has considered cases treated under the point-based treatment planning system, we recognize that centres are increasingly moving towards volume-based treatment planning, as well as combined intracavitary and interstitial brachytherapy systems. Under the GEC-ESTRO working group recommendations, high-risk (HR) CTV and intermediate-risk (IR) CTV areas should be delineated; the high-risk area receiving a total dose of greater than 80 Gy from EBRT and brachytherapy combined, and the intermediate-risk area receiving a total dose of at least 60 Gy. Ideally, an MRI should be obtained with each brachytherapy fraction, and the target should be modified with each fraction $[20,21]$. With volume based planning, we would expect the doses received by the PLNs to be influenced by the volumes of the HR and IR CTVs, potentially resulting in more inter-patient variation depending on anatomical differences as well as extent of disease.

Meanwhile, the use of interstitial needles has also become more common, especially in patients with parametrial invasion where intracavitary applicators alone may not be able to delivery sufficient dose [22]. These advances in treatment planning and technique have the advantage of increasing $\mathrm{D}_{90}$ of the target volume while reducing $\mathrm{D}_{2 \mathrm{cc}}$ of OARs. When interstitial needles are used, we would expect the doses to the PLNs to increase due to anatomical proximity. However at present, there is a paucity of data exploring BT dose to pelvic nodal regions in volume based and interstitial brachytherapy, hence it is difficult to quantify the extent of which dose to the various PLN groups would be affected by these new techniques.

In summary, nodal regions are complex and dosimetrics to specific nodes are dependent on various factors such as location, size, and movement of nodes. In this study, we have calculated the mean doses received to each nodal group as a contribution from intracavitary brachytherapy. We have also demonstrated the variation of doses within each nodal region as well as among our patient population. We believe that this information not only adds to and validates the data from the few published studies on this subject, but at the same time provides useful reference for the radiation oncologist during external beam planning to estimate the boost dose required, based on the location of the PLN within the pelvis.

\section{Conclusions}

Our study demonstrates that pelvic lymph nodes receive a significant dose contribution from HDR intracavitary brachytherapy in the radical treatment of cervical cancer. The dosimetric results presented in this paper serve as a guide for the radiation oncologist to estimate doses received by involved lymph nodes from brachytherapy, and to take them into account during the prior external beam radiotherapy planning phase.

\section{Acknowledgements}

We would like to express our gratitude to Nurun Nisa Amatullah for her advice regarding presentation of data. 


\section{Disclosure}

Authors reports no conflict of interest.

\section{References}

1. Srivastava A, Datta NR. Brachytherapy in cancer cervix: time to move ahead from point A? World J Clin Oncol 2014; 5: 764-774.

2. Lee LJ, Sadow CA, Russell A et al. Correlation of Point B and Lymph Node Dose in 3D-Planned High-Dose-Rate Cervical Cancer Brachytherapy. Int J Radiat Oncol Biol Phys 2009; 75: 803-809.

3. Lee Y, Rash DL, Stern RL et al. Variation in High-Dose-Rate Brachytherapy Dose Contribution Among Pelvic Lymph Node Groups in Locally Advanced Cervical Cancer. Int J Radiat Oncol Biol Phys 2014; 90: S47.

4. Mohamed SM, Aagaard T, Fokdal LU et al. Assessment of radiation doses to the para-aortic, pelvic, and inguinal lymph nodes delivered by image-guided adaptive brachytherapy in locally advanced cervical cancer. Brachytherapy 2015; 14: 56-61.

5. Van de Kamer J, De Leeuw A, Schippers M et al. Dosimetric interaction between lymph node irradiation and MR based brachytherapy for advanced cervical cancer. Radiother Oncol 2011; 99: S1-S259.

6. Taylor A, Rockally AG, Powell ME. An Atlas of the Pelvic Lymph Node Regions to Aid Radiotherapy Target Volume Definition. Clin Oncol (R Coll Radiol) 2007; 19: 542-550.

7. Toita T, Ohno T, Kaneyasu Y et al. A Consensus-based Guideline Defining Clinical Target Volume for Primary Disease in External Beam Radiotherapy for Intact Uterine Cervical Cancer. Jpn J Clin Oncol 2011; 41: 1119-1126.

8. Small W Jr, Mell LK, Anderson P et al. Consensus guidelines for delineation of clinical target volume for intensity-modulated pelvic radiotherapy in postoperative treatment of endometrial and cervical cancer. Int J Radiat Oncol Biol Phys 2008; 71: 428-434.

9. Lim K, Small W Jr, Portelance L et al. Consensus guidelines for delineation of clinical target volume for intensity-modulated pelvic radiotherapy for the definitive treatment of cervix cancer. Int J Radiat Oncol Biol Phys 2011; 79: 348-355.

10. Hoyte L, Thomas J, Foster RT et al. Racial differences in pelvic morphology among asymptomatic nulliparous women as seen on three-dimensional magnetic resonance images. Am J Obstet Gynecol 2005; 193: 2035-2040.

11. Wakatsuki M, Ohno T, Kato S et al. Impact of boost irradiation on pelvic lymph node control in patients with cervical cancer. J Radiat Res 2014; 55: 139-145.

12. Grigsby PW, Singh AK, Siegel BA et al. Lymph node control in cervical cancer. Int J Radiat Oncol Biol Phys 2004; 59: 706-712.

13. Ariga T, Toita T, Kasuya G et al. External beam boost irradiation for clinically positive pelvic nodes in patients with uterine cervical cancer. J Radiat Res 2013; 54: 690-696.

14. Nishimura Y, Komaki R. Intensity-Modulated Radiation Therapy: Clinical Evidence and Techniques. Springer Press, Japan 2015.

15. National Comprehensive Cancer Network. Cervical Cancer (version 1.2015). Available from: www.nccn.org/professionals/physician_gls/PDF/cervical.pdf. Accessed 15.03.2016.

16. Abe T, Tamaki T, Makino $S$ et al. Assessing cumulative dose distributions in combined radiotherapy for cervical cancer using deformable image registration with pre-imaging preparations. Radiat Oncol 2014; 9: 293.

17. Van de Kamer JB, De Leeuw AA, Moerland MA et al. Determining DVH parameters for combined external beam and brachytherapy treatment: 3D biological dose adding for patients with cervical cancer. Radiother Oncol 2010; 94: 248-253.
18. Hinton B, Fiveash $\mathrm{J}, \mathrm{Wu} \mathrm{X}$ et al. Optimal planning target volume margins for elective pelvic lymphatic radiotherapy in high-risk prostate cancer patients. ISRN Oncology 2013; 2013: 941269.

19. Ramlov A, Kroon PS, Jürgenliemk-Schulz IM et al. Impact of radiation dose and standardized uptake value of (18)FDG PET on nodal control in locally advanced cervical cancer. Acta Oncol 2015; 54: 1567-1573.

20. Pötter R, Haie-Meder C, Van Limbergen E et al. Recommendations from gynaecological (GYN) GEC ESTRO working group (II): Concepts and terms in 3D image-based treatment planning in cervix cancer brachytherapy-3D dose volume parameters and aspects of 3D image-based anatomy, radiation physics, radiobiology. Radiother Oncol 2006; 78: 67-77.

21. Banerjee R, Kamrava M. Brachytherapy in the treatment of cervical cancer: a review. Int J Womens Health 2014; 6: 555-564.

22. Otter S, Franklin A, Ajaz M et al. Improving the efficiency of image guided brachytherapy in cervical cancer. J Contemp Brachytherapy 2016; 8: 557-565.

23. Population Trends 2015, Dept of Statistics Singapore. Available from: https://www.singstat.gov.sg/docs/default-source/ default-document-library/publications/publications_and_ papers/population_and_population_structure/population 2015.pdf. Accessed 10.04.2016. 